\title{
Biaxial Deformation in High Purity Aluminum
}

V. Livescu ${ }^{1}$, J.F. Bingert ${ }^{2}$, C. Liu ${ }^{1}$, M.L. Lovato ${ }^{1}$, B.M. Patterson ${ }^{1}$

${ }^{1}$ Los Alamos National Laboratory, Materials Science and Technology Division, Los Alamos, NM 87545

${ }^{2}$ OUSD(AT\&L)/TWS/LW\&M, Washington, D.C. 20301, USA

Corresponding author: Veronica Livescu, vlivescu@lanl.gov, Phone: 505-667-7537, Fax: 505-

667-8021, Mail Stop G755, Los Alamos, NM87545

\section{Abstract}

The convergence of multiple characterization tools has been applied to investigate the relationship of microstructure on damage evolution in high purity aluminum. The extremely coarse grain size of the disc-shaped sample provided a quasi-two dimensional structure from which the location of surface-measured features could be inferred. In particular, the role of preexisting defects on damage growth was accessible due to the presence of casting porosity in the aluminum. Micro tomography, electron backscatter diffraction, and digital image correlation were applied to interrogate the sample in three dimensions. A recently developed micro-bulge testing apparatus was used to deform the pre-characterized disc of aluminum in biaxial tension, and related analysis techniques were applied to map local strain fields. Subsequent post mortem characterization of the failed sample was performed to correlate structure to damaged regions. It was determined that strain localization and associated damage was most strongly correlated with grain boundary intersections and plastic anisotropy gradients between grains. Pre-existing voids played less of an apparent role than was perhaps initially expected. These combined techniques 
provide insight to the mechanism of damage initiation, propagation, and failure, along with a test bed for predictive damage models incorporating anisotropic microstructural effects.

Keywords: aluminum, plasticity, digital image correlation, damage localization, bulge test

\section{Introduction}

There are a number of service environments that expose structural metals to extremes of stress, strain rates, and strains. These include aerospace, automotive, manufacturing, and military applications. It is desirable to predict the response of structural metals in these extreme environments and under complex loading configurations that components may see in applications in order to eventually create more damage-tolerant designs. Therefore, a long-term

goal is the development of physically-based and process-aware modeling capabilities targeted to simulate high-rate, large-strain deformation and damage scenarios.

Existing models, by their nature, homogenize the natural length scales via polycrystalline averaging. The major weakness of current modeling capabilities that simulate material damage and failure is the lack of proper physical representation of the damage mechanism and its relationship with the metal's microstructure, evolving statistical processes, and natural material length scales inherent within the material structure. Many researchers have stated the limitations of the numerical framework for predicting damage phenomena [1-3]. Based on experimental evidence, the damage nucleation process is believed to depend on microstructural-based heterogeneities and the spatial distribution of defects. The interplay among the process-derived heterogeneous microstructure, the defects, deformation-induced features, and failure mechanisms is very complex. It is thought that the statistical spatial distribution of inherent or deformation- 
induced heterogeneities act in combination with the spatial and temporal intensity of loading to determine which of the weakest defect sites will initiate a void [4-9]. In particular, there is no available physically-based continuum theory of damage that accounts for the effects of slip and texture development, void formation, and adiabatic heating. Since these are the main mechanisms responsible for the localization of deformation and failure, a damage theory incorporating all these effects in a direct and explicit manner is desirable.

Although deformation bands, shear bands, and twins have been studied in numerous microscopical investigations, their origin, evolution, and role during plastic deformation are not well understood. Dislocations and their mutual interactions determine the material strength in the absence of other internal defects and they organize in the form of patterns, resulting into a heterogeneous field of deformation at the micro scale. As a result, localized deformation, shear banding, and dislocation cell structures play a significant role in determining the flow properties of heterogeneous materials even at small macroscopic strains $[10,11]$. The plastic deformation follows a three stage process which begins with a general, uniform deformation with a homogeneous strain state, during which a density of statistically-stored dislocations accumulate. Homogeneous deformation of a polycrystalline aggregate requires that different grains deform in a compatible way. As deformation proceeds, larger misorientations develop in the substructure and the texture evolution becomes more sensitive to the character of the dislocation structure produced in a material. At this stage local, non-uniform deformation takes place, exhibiting an inhomogeneous strain distribution during which a density of geometrically necessary dislocations accumulate. Finally, localization into a fine shear band may occur, and this localization damage typically leads to eventual failure. At greater rates, adiabatic shear banding has also been viewed as a leading mechanism in the formation of micro cracks $[12,13]$. 
However, establishing the onset of failure in ductile metals remains a challenge. As a consequence, it is desirable to find ways to quantify microstructural heterogeneity to better inform multi-scale damage models.

To enable the prediction of deformation and failure in complex loading environments, such material models must be developed and validated through coordinated experimentation and theoretical approaches. The development of an enhanced predictive damage and failure capability requires improved understanding of the interaction between material microstructures and the imposed stress state. The emergence of advanced characterization capabilities has enabled the collection of unprecedented levels of data on the structural state of materials. The goal of this work is to leverage a group of these characterization tools to perform integrated 3D experiments under complex or integrated loading conditions that both validate and help improve predictive models. For example, experimental techniques that permit three-dimensional interrogation and in situ tracking of strain fields may help provide this insight. This work involves an approach that combines electron backscatter diffraction (EBSD), x-ray tomography, and a recently developed bulge testing technique [14] to enable the definitive correlation of strain localization with pre-existing microstructural features.

As a first step in this process, the objective is to determine how the process-induced microstructure drives the propensity for strain localization and determine relationships between microstructure and material failure that can be directly incorporated into models. The proposed experiment utilizes a multi-axial loading technique involving a miniature bulge test, along with in situ, three-dimensional digital image correlation (3D-DIC) diagnostics to examine the deformation and damage processes active during loading in high-purity aluminum. This technique enables the determination of 3D displacements and 2D planar strain fields on the upper 
surface of the deforming material bulge, followed by correlation of observed behavior to microstructural components for a specimen deformed to high strains but under moderate strain rates. The experiment is also a precursor to higher strain-rates or dynamic bulge tests that will provide information on large-strain deformation response of materials under high-rate loading.

This investigation revealed that a large deformation in the case of coarse-grained aluminum results in anisotropic and localized deformation along grain boundaries. Microstructural characteristics appear to have a stronger effect on the evolution of localization and damage in this large grained aluminum than the pre-existing process-generated defects.

\section{Materials and Methods}

The material used for this investigation is $99.99 \%$ high-purity cast aluminum with an analyzed chemical composition (wt. \%) of $15 \mathrm{ppm} \mathrm{Mg}, 7 \mathrm{ppm} \mathrm{Cu}, 10$ ppm Fe, 10 ppm Si, 5 ppm Zn, and balance aluminum. High-purity aluminum was chosen here for a number of reasons: (1) Importance as a model baseline material for understanding lightweight aluminum alloy systems relevant to many engineering applications; (2) The 2D nature of the coarse-grained microstructure enabled the interpolation of features such as grain boundaries to three dimensions. With regard to the last point, the microstructure of the as-received cast bar contains grains on the order of millimeters, exhibiting a columnar zone at the exterior and a small equiaxed zone in the center, typical of cast aluminum bars; (3) The aluminum bar stock exhibited casting porosity that provided a natural defect population of sufficient magnitude to explore the role of initiation sites on subsequent damage evolution, and; (4) As a low-atomic number metal with a low x-ray absorption coefficient, aluminum is conducive to radiographic volumetric characterization of the pre-deformed void population and the post-test damage state. 
The columnar grains that grew perpendicular to the casting mold provide an ideal morphology for this experiment. A $25.4 \mathrm{~mm}$ diameter by $1.2 \mathrm{~mm}$ thick disc was machined so that the disc thickness was perpendicular to the long-axis of the grains. The resulting dimensions captured a reasonable number of grains that extended through the thickness of the disc, thus a 3D specimen with a 2D microstructure. The disc was also of sufficient thickness to assure that the sample was impermeable, and thus could hold pressure for the subsequent hydraulic bulge test.

As mentioned in the introduction, the anisotropic character of materials is a factor in how localization damage develops. As a consequence, we investigate here the integrated role of both the crystallographic properties and the presence of pre-existing defects on the deformation behavior of aluminum during the miniature bulge test.

The surface of the high-purity aluminum disc described above was prepared using classic metallographic methods to obtain a mirror finish. The polished surface was then lightly scribed to demarcate the bulge test gage area, and a fiducial mark was made as an aid for subsequent alignment and registration. The microstructure of the polished disc was characterized on both sides by means of Electron Backscatter Diffraction (EBSD) and X-ray computed microtomography (XCMT). EBSD analysis was performed on a Phillips XL30 FEG SEM, using a voltage of $20 \mathrm{kV}$, a spot size of 4 , in stage scan mode, with a step size of 50 microns. Using stage-scan mode enabled EBSD characterization of the entire disc with a single scan, thus avoiding the need to stitch together several data files. The disc was also analyzed with an Xradia XCMT instrument to reveal the pre-existing defects in the material.

EBSD and XCMT data are presented in Figures 1a and 1b. In both of these images, the scribe line that demarcates the circular gage area is visible, along with a fiducial mark located adjacent to the gage scribe on the left side of the disc. Figure 1a provides crystallographic 
orientation information, along with the location of grain boundaries that transect the surface. Figure $1 \mathrm{~b}$ shows the superposition of EBSD-derived grain boundaries registered -with the XCMT data, where internal voids are highlighted in pink. In addition, the locations of surface voids observed following bulge testing are denoted with black circles. This figure indicates the location of porosity with respect to the grain boundary network. Post-mortem characterization of the aluminum bulge sample was performed using Stereo Optical Microscopy (SOM) and Scanning Electron Microscopy (SEM).

\section{Test Configuration for Miniature Bulge Test and 3D-DIC}

The technology we used to study the surface deformation of a thin aluminum round disc is the miniature bulge test combined with the optical technique of 3D-DIC. An overview of the miniature bulge test configuration is presented in Figure 2. The miniature bulge test essentially uses a thin sheet of sample material deformed into a bulge by applying pressure on one side of the sheet. The experiment was performed in air, at room temperature. Figure 2 presents details of the miniature bulge test specimen, the pressure cell used to pressurize the specimen, and the associated DIC and recording instrumentation [14].

The aluminum disc rests inside a custom-made cell that is pressurized by infusing distilled water using a PHD ULTRA 4400 syringe pump from Harvard Instruments. This is a very high precision syringe pump that allows variable infusion rates. The syringe pump serves as the loading device to apply mechanical load to the specimen, while the pressure cell is connected to a pressure gage. 
To capture and monitor the evolving deformation field on a curved three-dimensional surface, or the out-of-plane motion on a planar sample surface, we use the two-camera system. The two CCD cameras are positioned above the testing specimen and they capture a series of paired images of the specimen during the test. Other details of the test system and its calibration procedure are described by Liu [14]. The pressure gage and the two CCD cameras are connected to a computer, so that the same computer captures both the pressure signal and the digital images synchronized in time. Detailed description of the underlying principles of the DIC technique can be found in the literature, e.g., [15]. In order to apply the 3D-DIC technique, a random speckle pattern was applied to the specimen surface by first spraying a very thin layer of white paint as a background and then spraying black paint on top of the background to generate a speckle pattern. During the bulge test, a series of image pairs of the random speckle pattern was acquired by the two CCD cameras. Analysis of the 3D-DIC data allows calculation of the 3D displacements and 2D planar strain fields on the upper surface of the bulge.

\section{Results}

The aluminum test specimen was characterized prior to and after testing. Here, results from post mortem characterization and in situ 3D-DIC diagnostics are discussed so as to correlate the initial microstructure with the evolving material damage and associated mechanical response under integrated loading conditions. In what follows, the metallography and in situ measured strain fields will be discussed. Correlations between microstructural features, strain fields, and damage are drawn based on connections between pre-test characterization, post-mortem characterization, and 3D-DIC data analysis, as described in the following sections. 


\section{Post-mortem sample characterization}

A montage of surface damage features from the post mortem sample is shown in Figure 3. A stereo microscopy image of the recovered sample is shown in the center; circles indicate the surface damage locations. This stereo image is surrounded by higher magnification SEM images of the surface damage, with the image boundaries color-coded to the circles. In the common cases of static or quasi-static hydraulic loading the pressure distribution on the tested specimen is uniform and the bulge is developed with a nearly hemispherical shape. In this case, the aluminum disc deviates slightly from a spherical shape, as the disc's deformation becomes heterogeneous as the test progresses. The surface of the dome is faceted due to the local deformation characteristics imposed by individual through-thickness grains, essentially producing a severe manifestation of the orange peel effect commonly encountered in the deformation of coarse-grained sheet.

The post-mortem analysis of the recovered aluminum test specimen is focused on connecting the surface damage observed in Figure 3, along with the localized plasticity determined through the 3D-DIC, to the pre-test microstructure presented in Figure 1, including pre-existing porosity introduced through the manufacturing process. A few of these voids are spatially associated with boundaries, but many are not. By analyzing the location of deformation induced damage with respect to the grain boundary map and the orientation information we can ascertain the relative influence of voids, grain boundaries, and plastic anisotropy in the damage nucleation and evolution process. 
SEM analysis was performed on the outer surface of the bulged specimen. Voids observed on the specimen surface after the tests are shown in black in Figure 1b. SEM images of the voids found on the bulge surface are shown in Figure 3. Deformation-induced voids are formed in connection to grain boundaries or triple points. This implies that the neighboring crystals interact under loading and the effect of such interaction could depend on their mutual crystallographic orientation.

To examine the potential role of orientation and anisotropy further, the Taylor theory for polycrystalline plasticity can be employed. The Taylor factor map for the polycrystalline aluminum disc is shown in Figure 4. The map was constructed from the EBSD scan assuming an imposed biaxial strain in the plane of the grains. The Taylor factor map quantifies a grain's relative propensity for slip based on its orientation, active slip systems, and the imposed strain gradient. Specifically, in Figure 4, grains that are blue in color are most favorably oriented for slip, while grains that are red in color are least favorably oriented. Furthermore, sharp differences in Taylor factor across grain boundaries may be an indication of strain incompatibility leading to local stress concentrations. Additionally, it has been shown by Lebensohn [16] that grains in 'hard' orientations for deformation (red grains) can mitigate void growth. Frequently, deformation-induced voids are found at triple junctions where low and high Taylor factor grains meet, as indicated by the darker green, red, yellow and light blue circles in Figure 4. Actual sample failure occurred at the location shown by the red circle, where failure along boundaries was severe enough to penetrate all the way through the disc thickness and cause an abrupt drop in water pressure recorded at the end of the experiment.

Only a few of the pre-existing voids were observed in the post-mortem SEM analysis of the sample surface, the remaining ones are deeper in the thickness of the disc and were observed 
only in the XCMT examination. These top surface intra-granular voids appear virtually unaffected after the test.

\section{In situ 3D-DIC Characterization}

Figure 5 shows a pair of the speckle images, from the left and the right CCD cameras, acquired at two different moments during the test of the disc. A circular bulge can be seen at the center of the image. As the applied pressure increases, the bulge continuously enlarges. At each instant of time during the deformation, the 3-dimensional profile of the sample surface is quantified based on the evolution of speckle displacement during bulging. The evolution of the shape of the bulge can be obtained, and consequently, evolution of displacement field on the sample surface is determined. From the displacement field and the geometric profile of the evolving bulge during the progression of the test, the strain field and the curvature field along the bulge surface can be calculated.

\section{D-DIC data analysis}

Based on the relative orientations of the stereo cameras and internal camera parameters, the 3D-DIC allows measurements such as evolution of disc displacement in three directions, and the associated applied pressure at any given moment. The evolution of displacement $(u, v, w)$ at any location within the $\mathrm{Al}$ specimen and the associated applied pressure $(\mathrm{P})$ as a function of time are shown in Figure 6. Subject to low pressure, $\mathrm{P}<1.75 \mathrm{MPa}$, the aluminum sample deforms linearly. As the pressure exceeds 1.75MPa, the aluminum sample exhibits yield-like behavior. The yielding point corresponds to a deflection of approximately $0.04 \mathrm{~mm}$. At moment $\mathrm{F}$, the applied pressure $\mathrm{P}$ reaches its maximum of $11.7 \mathrm{MPa}$, after which the applied pressure suddenly drops due to sample failure and the test is stopped. 
By repeating the process at every instant of time during the test, the evolution of the shape of the bulge can be obtained, and consequently, evolution of the displacement field on the sample surface is determined. At each time step during the deformation, the 3-dimensional profile of the sample surface is quantified. For times A-F shown in the plot in Figure 6, the elevation $w$ of the bulge is mapped. The maximum vertical deflection recorded by 3D-DIC is $4.2 \mathrm{~mm}$. This value agrees well with the post-mortem deflection of $4.3 \mathrm{~mm}$ measured directly on the recovered specimen. The values for $u$ and $v$ are also available. Having the evolution of $u, v$, and $w$, we can reconstruct the top surface of the disk and its evolution with time is reconstructed. In this way, the 3D-DIC data provides information about the axisymmetry of the developing bulge for each point in Figure 6. The plot in Figure 7 describes the average bulge deflection as a function of radius. Starting from the center of the disc and with a radius $r$, we compute the average $w$ and the standard deviation (shown as the error bar) is computed. The radius $r$ is extended to the boundary of the field of view. Up to moment $\mathrm{D}$, the bulging deformation of the $\mathrm{Al}$ disc is quite axisymmetric. However, at moments $\mathrm{E}$ and $\mathrm{F}$, the error bars become larger, indicating that the bulging of the disc is somewhat deviating from axisymmetry. This result agrees well with the post-mortem observations. The deviation from axisymmetry correlates to the grains' anisotropic deformation.

Once the 3D contour has been determined, the next step in 3D-DIC is the determination of the three-dimensional deformation of the object surface. This process is performed by correlation of the images, taken by both cameras in the deformed state, with their original reference images. From the displacement field and the geometric profile of the evolving bulge during the process of test, the strain field and the curvature field along the bulge surface can be 
calculated. A Cartesian coordinate system is defined such that $(x, y)$ is a flat planar surface. The third axis $z$ is perpendicular to the $x-y$ plane and in the direction of the bulge deflection.

For the aluminum disc tested here, the maximum deflection of $4.2 \mathrm{~mm}$ is much larger than the disc thickness of $1.2 \mathrm{~mm}$. In addition, the ratio between the initial diameter of the aluminum disc and its thickness is about 10.6, which is not large enough so that membrane theory alone can accurately describe the deformation of the bulge. As a result, a combination of the membrane theory and the plate theory is required.

Since there are no shear strain components in this coordinate system, the principal strains represent the maximum and minimum stretches of an elemental volume. Figure 8 shows maps of the major and minor principal strain fields and maps of the orientation of major principal strain with respect to the $\mathrm{x}$-axis at times A, C, D, E, and F during the test. Figure 8 shows that the principal strains are mostly tensile in nature and increase with loading to values as high as $40 \%$. The only compressive strains are found around the edges of the bulge where geometric curvature is pronounced due to the restrain imposed by the disc holder. The principal strains are uniform in the early stages of deformation as shown in the maps for times A, B, and C. As the aluminum sample deforms and develops into a bulge, the major and minor strain maps develop into localizations. The first increase in strain values is observed at time C. As the bulge evolves, the strain affected region expands around the center of the disc. The highest values for the major principal strain are found at triple points. These triple points are known to play an important role in a polycrystal deformation. Randle [17] has shown that, under large deformation, the level of grain orientation perturbations increases with strain and that enhanced zones of perturbations are observed at grain boundaries and especially near triple junctions. In addition, high values in 
major principal strains develop intragranularly in grains exhibiting a lower Taylor factor value that are surrounded by higher Taylor factor grains. For example, the voids indicated by the turquoise, red, yellow, purple and light-blue circles in Figure 3 occurred at boundaries between a low and high Taylor factor grains. These grains have a lower propensity to slip, but they must accommodate deformation gradients imposed by the surrounding more slip-prone grains.

The maps of the orientation of major principal strain with respect to the $\mathrm{x}$-axis reflect sharp changes in major principal strain direction. Such sharp gradients are associated with plastic anisotropy and are spatially correlated with grain boundary regions. They may result in damage accumulation at boundaries due to strain incompatibility.

The mean curvature characterizes the degree of bending of a surface. The maximum and minimum of the normal curvature at a given point on a surface are called principal curvatures. The principal curvatures measure the maximum and minimum bending of a regular surface at each point. Figure 9 contains the principal curvature maps and the orientation of the major principal curvature. Sudden changes of curvature orientation are utilized to indicate connection to microstructural features or regions with different deformation characteristics. Positive curvature values indicate development of a convex region, while negative curvature indicates the development of a concave region on the bulge surface. Evidently, a curvature value of zero is indicative of a flat surface. High values of major and minor curvature are evident from the beginning of the experiment around the base of the bulge. As the bulge grows, principal curvatures begin to develop local peaks. Peaks in major principal curvature are intragranular, witnesses of grains' accommodation response to different strains from other neighboring grains. The minor principal curvature field exhibits also localized peaks, but in this case they occur in the immediate vicinity of grain boundaries. Due to the stretching effect imposed by the 
increasing bulge and before compromising the grain boundary cohesion, the interior of the grain undergoes plastic deformation to equilibrate the neighboring stress state. The localized increase in the minor principal curvature begins around time $\mathrm{C}$, similar to the principal strains, at triple junctions or along boundaries between grains with low and high Taylor factors. As deformation evolves, more boundaries are affected.

\section{Discussion}

Several potential microstructural features were considered as influential in the damage evolution process, and characterized by EBSD, XCMT, SEM, and 3D-DIC. These characteristics consisted of the grain boundary network, plastic anisotropy, void location, and strain localization. In order to tie these together, the maps in Figure 10 were constructed from the previously discussed data. The region with the largest magnitude of the major principal strain field at moment $\mathrm{E}$ was segmented from the map in Figure 8. This region of the most intense strain localization was superimposed on the Taylor factor map, as shown in Figure 10a. The localized plasticity field is roughly correlated with the blue, softest orientation, grains near the center of the sample. However, significant deviations are observed. It is clear that orientation alone is not controlling the localization response, or a band running diagonally across the blue grain region would be expected. Consideration of the grain boundary network provides additional insight. The tail of the localization region, although running out of the soft grains into the orange-red grains, is aligned near a grain boundary separating the two hard grains. Although not perfectly aligned, it should be noted that the inclination of the boundary into the plane of the sample is unknown, so the localization may more closely follow the internal boundary. In addition, the smaller island of localization to the right of the main region lies both within soft grains and along a boundary. Referring back to Figure 8, the localization appears to be nucleated 
at or near to what is close to a vertex, or confluence of four grains at a point. The growth of this region then favors boundaries and soft grains.

As discussed in the introduction, more evidence is being brought by research to support what was observed in many instances: the characteristics of the materials' internal structure play a critical role in the deformation and failure of a material under loading. Several microstructure characteristics have been identified as potential factors for material failure: distribution of grain boundary types, presence of preferred orientation, distribution of grain size, and density of defects and impurities. This study was designed to help us link microstructure to deformation and/or failure in a large grained high-purity aluminum. In the absence of other internal impurities, the evolution of the microstructure under large plastic deformation results into a heterogeneous field of deformation at the micro scale. A high-purity aluminum disc was subjected to biaxial loading in a miniature bulge test and characterized in-situ using 3D-DIC. Insitu strains were mapped, so that the developing incompatibilities can be observed. We learned that damage under biaxial loading produced in a bulge test is associated with certain neighborhoods of grains. More specifically, void damage occurs due to strain incompatibilities at boundaries were there is need to accommodate deformation gradients imposed by the surrounding more slip-prone grains. In three instances voids observed post-mortem by SEM corresponded to voids observed by XCMT. However, these voids did not grow or evolve significantly.

\section{Conclusions}

A technique that combines the miniature bulge test and the 3D-DIC is implemented for quantitatively measuring the stretching and bending in a high-purity, large grained aluminum disc. EBSD, XCMT, 3D-DIC, hydraulic bulge testing were combined for detailed 
characterization of the bulge-induced deformation of a high-purity aluminum disc. The findings can be summarized as follows:

- 3D-DIC provides the time evolution of 3D displacements and 2D planar strain fields on the upper surface of an aluminum specimen following a hydraulically drive bulge test.

- At large deformations, the bulge of the large-grained aluminum disc deviates from axisymmetry and exhibits anisotropic and localized deformation along grain boundaries. Thus study indicates that triple and quadruple junctions, as well as sharp differences in Taylor factor values play a critical role in the development of plastic localization and damage.

- The highest values for the major principal strain are found at triple and quadruple points.

- Microstructural characteristics appear to have a stronger effect on the evolution of localization and damage in this large grained aluminum than the pre-existing porosity.

These conclusions suggest that for damage evolution and failure models to accurately capture and predict the evolution of deformation and damage processes that incorporate shear localization, banding and failure, they cannot merely include a critical stress for failure. Instead, models must account for the relationship between the microstructural evolution and the applied and evolving integrated stress states during a loading event.

\section{Acknowledgements}

Los Alamos National Laboratory is operated by LANS, LLC, for the NNSA of the US Department of Energy under contract DE-AC52-06NA25396. This work has been performed under the auspices of the United States Department of Energy and was supported by the Joint Department of Defense (DoD) and Department of Energy (DOE) Munitions Technology 
Development Program. We thank Michael FitzGibbon, US Naval Academy and LANL Service Academy Research Associate, for his insight and assistance with data analysis.

\section{References}

1. W.F. Hosford and W.A. Backofen, 1962, "Strength and plasticity of textured metals", 9th Sagamore Ordnance Materials Research Conference.

2. W. T. Lankford, S. C. Snyder, J. A. Bauscher, 1950, "New criteria for predicting the press performance of deep drawing sheets", Transactions American Society for Metals, $42,1197$.

3. D. Steglich, Y. Jeong, M.O. Andar, T. Kuwabara, 2012, "Biaxial Deformation Behavior of AZ31 Magnesium Alloy: Crystal-Plasticity-Based Prediction and Experimental Validation”, International Journal of Solids and Structures, 49(25), 3551-3561.

4. Adams, B. L., Olson, T., 1998, "The microstructure properties linkage in polycrystals", Progress in Materials Science, 42, 1-87.

5. E.K. Cerreta, I.J. Frank, G.T. Gray III, et al., 2009, "The influence of microstructure on the mechanical response of copper in shear", Materials Science and Engineering AStructural Materials Properties Microstructure and Processing, 501(1-2), 207-219.

6. J.P. Escobedo, E.K. Cerreta, D. Dennis-Koller, et al., 2008, “ Effects of microstructure and shock loading conditions on the damage behavior of polycrystalline copper", EPJ Web of Conferences, 26, 02008.

7. Livescu, V., Bingert, J. F., Mason, T. A., 2012,'Deformation twinning in explosivelydriven tantalum", Materials Science and Engineering A-Structural Materials Properties Microstructure and Processing, 556, 155-163.

8. N.A. Pedrazas, D.L. Worthington, D.A. Dalton, et al., 2012, "Effects of microstructure and composition on spall fracture in aluminum", Materials Science and Engineering AStructural Materials Properties Microstructure And Processing, 536, 117-123.

9. P. B. Trivedi, J. R. Asay, Y. M. Gupta, and D. P. Field, 2007, "Influence of grain size on the tensile response of aluminum under plate-impact loading", Journal of Applied Physics, 102, 083513.

10. S.V. Kamat and J.P. Hirth, 1990, "Dislocation injection in strained multilayer structures", Journal of Applied Physics, 67, 6844-6850. 
11. M. Rhee, J.P. Hirth, and H.M. Zbib, 1994, “A Superdislocation Model for the Strengthening of Metal Matrix Composites and the Initiation and Propagation of Shear Bands", Acta Metallurgica et Materialia, 42(8), 2645-2655.

12. H.M. Zbib and J.S. Jubran, 1992, "Dynamic Shear Banding: A Three-Dimensional Analysis", International Journal of Plasticity, 8(6), 619-641.

13. J. Lambros and A.J. Rosakis, 1995, "Shear Dominated Transonic Interfacial Crack Growth in a Bimaterial-I. Experimental Observations", Journal of the Mechanics and Physics of Solids, 43(2), 169-188.

14. C. Liu, K. Clarke, K. Hollis, M. Lovato, D. Alexander, W. Blumenthal, and D. Dombrowski, 2014, "Measuring Fracture Toughness in LEU Fuel Plates Containing Al/Zr/DU-10 wt\% Mo Interfaces Using Miniature Bulge Test \& 3D-DIC", Los Alamos National Laboratory Report LA-UR-14-24547, p 5-10.

15. M.A. Sutton, S.R. McNeill, J.D. Helm, and Y.J. Chao, 2000, "Advances in twodimensional and three-dimensional computer vision”, Topics in Applied Physics, 77, 323-372.

16. R.A. Lebensohn, J.P. Escobedo, E.K. Cerreta, et al. 2013, "Modeling void growth in polycrystalline materials", Acta Materialia, 61(18), 6918-6932.

17. V. Randle, N. Hansen, D.J. Jensen, 1996, “The Deformation Behavior of Grain Boundary Regions Polycrystalline Aluminium”, Philosophical Magazine A-Physics of Condensed Matter Structure Defects and Mechanical Properties, 73(2), 265-282. 


\section{List of Figure Captions:}

Figure 1: Pre-test sample characterization indicating a) crystal direction map and b) presence of pre-existing voids (XCMT data) shown in pink.

Figure 2: Experimental set-up for miniature bulge test and 3D-DIC.

Figure 3: SEM images of surface damage observed in the aluminum sample after testing.

Figure 4: Taylor factor map of bulge specimen in pre-tested condition, for biaxial deformation in plane of sheet and fcc restricted glide. Lower Taylor factors correspond to grains oriented more favorably for plastic deformation.

Figure 5: Typical pairs of random speckle images acquired from the left and the right CCD cameras, respectively, during the controlled-bulge test of the aluminum/aluminum sample.

Figure 6: Evolution of the bulge in the aluminum at selected moments of time during the miniature bulge test. The associated applied pressure at each instant of time is also shown.

Figure 7: Average disc deflection, obtained from the 3D-DIC data for time steps A-F shown in Figure 4.

Figure 8: Maps of major and minor principal strain fields, together with orientations of major principal strain (theta with respect to $\mathrm{X}$-axis).

Figure 9: Maps of major and minor curvature fields, together with orientations of major principal curvature ( $\gamma$ with respect to $\mathrm{X}$-axis).

Figure 10: Superimposed images of a) Taylor factor map and outline of greatest principal strain, and b) grain boundary map, maximum principal strain zone, pre-existing porosity (pink), and post mortem surface voids (blue). 
Figures

Figure 1
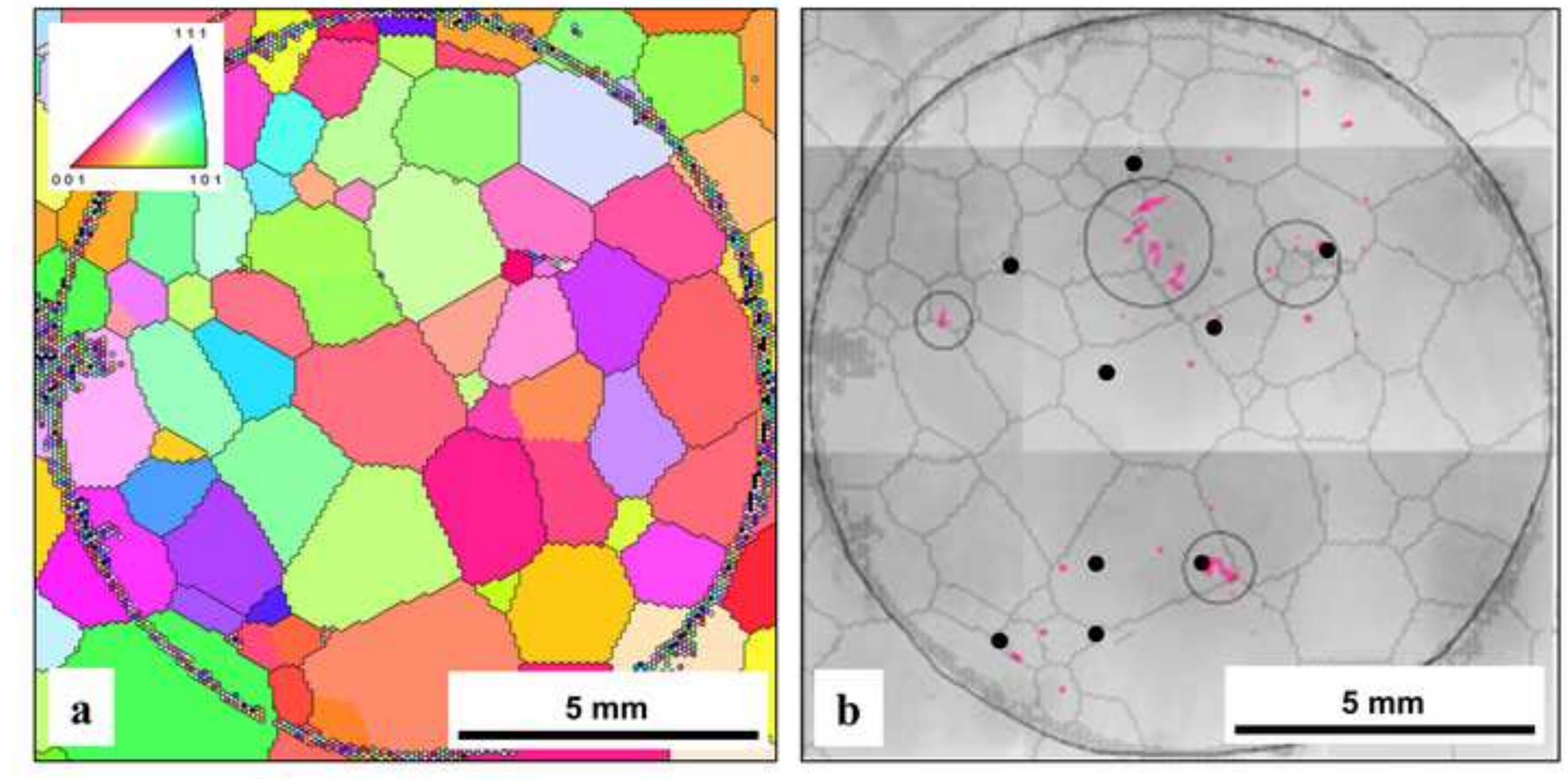

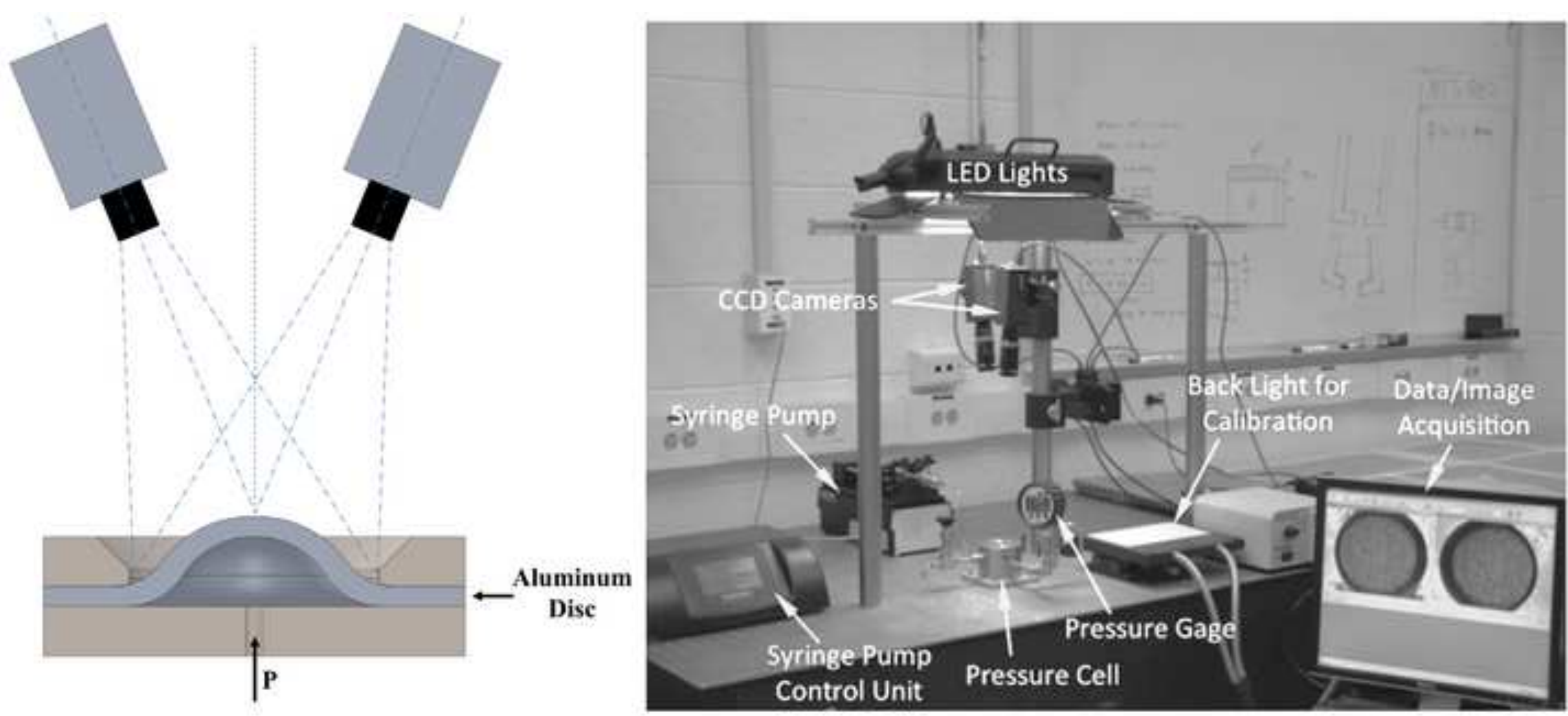

luminum

Dise Control Unit 

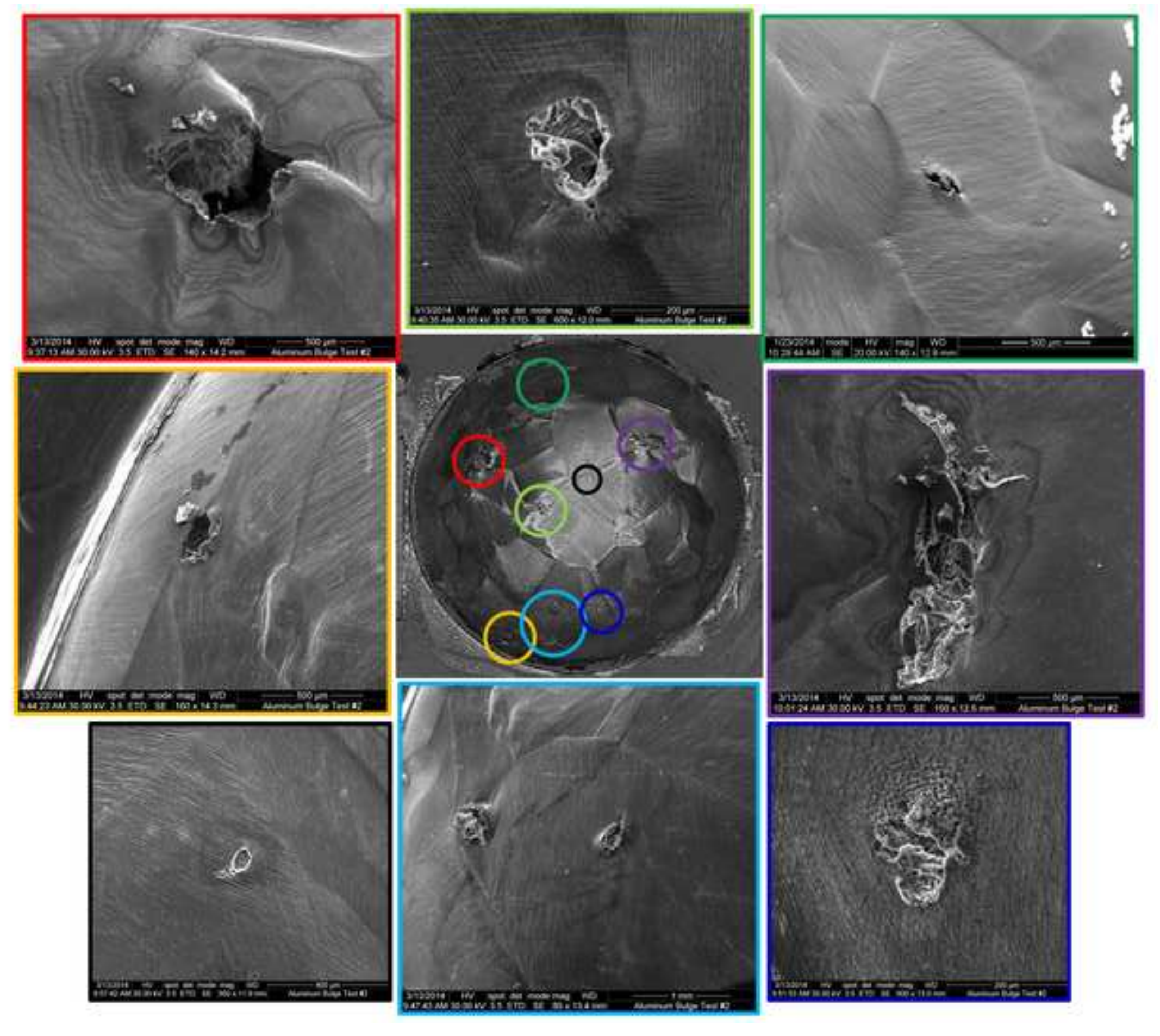

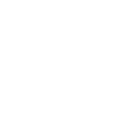



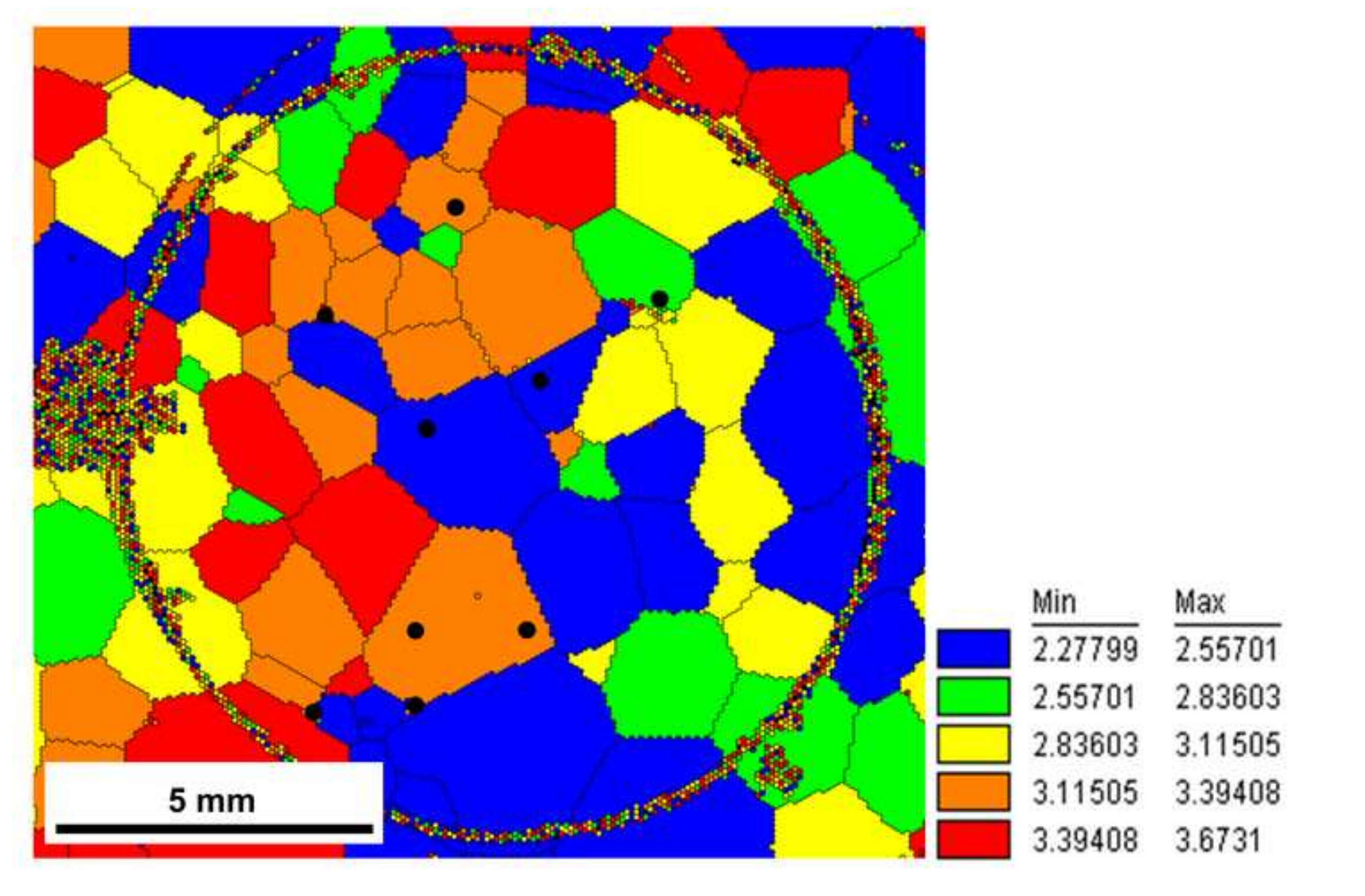

列

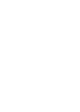

.



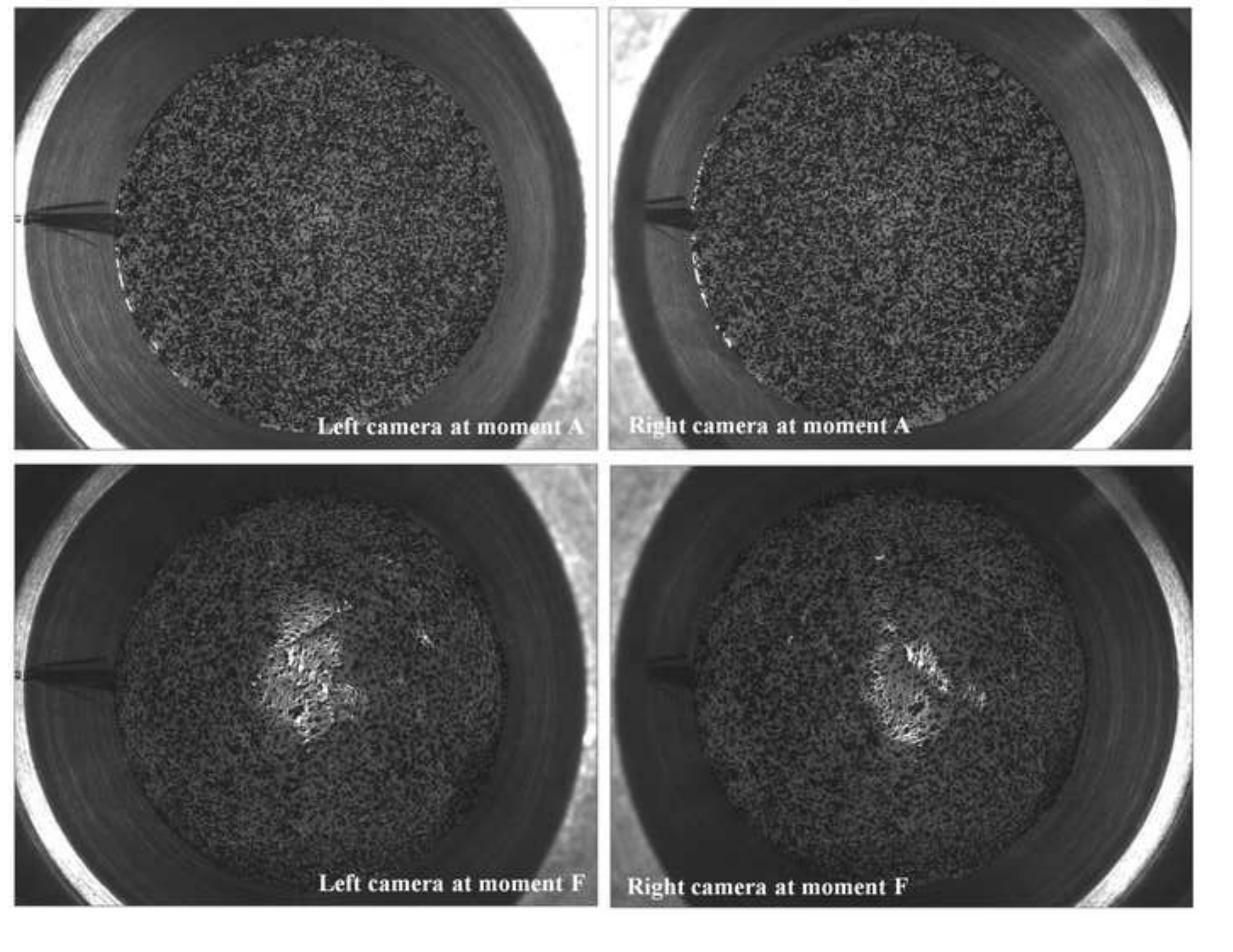

ayseft camera at moment A

Right camera at moment $A$ 


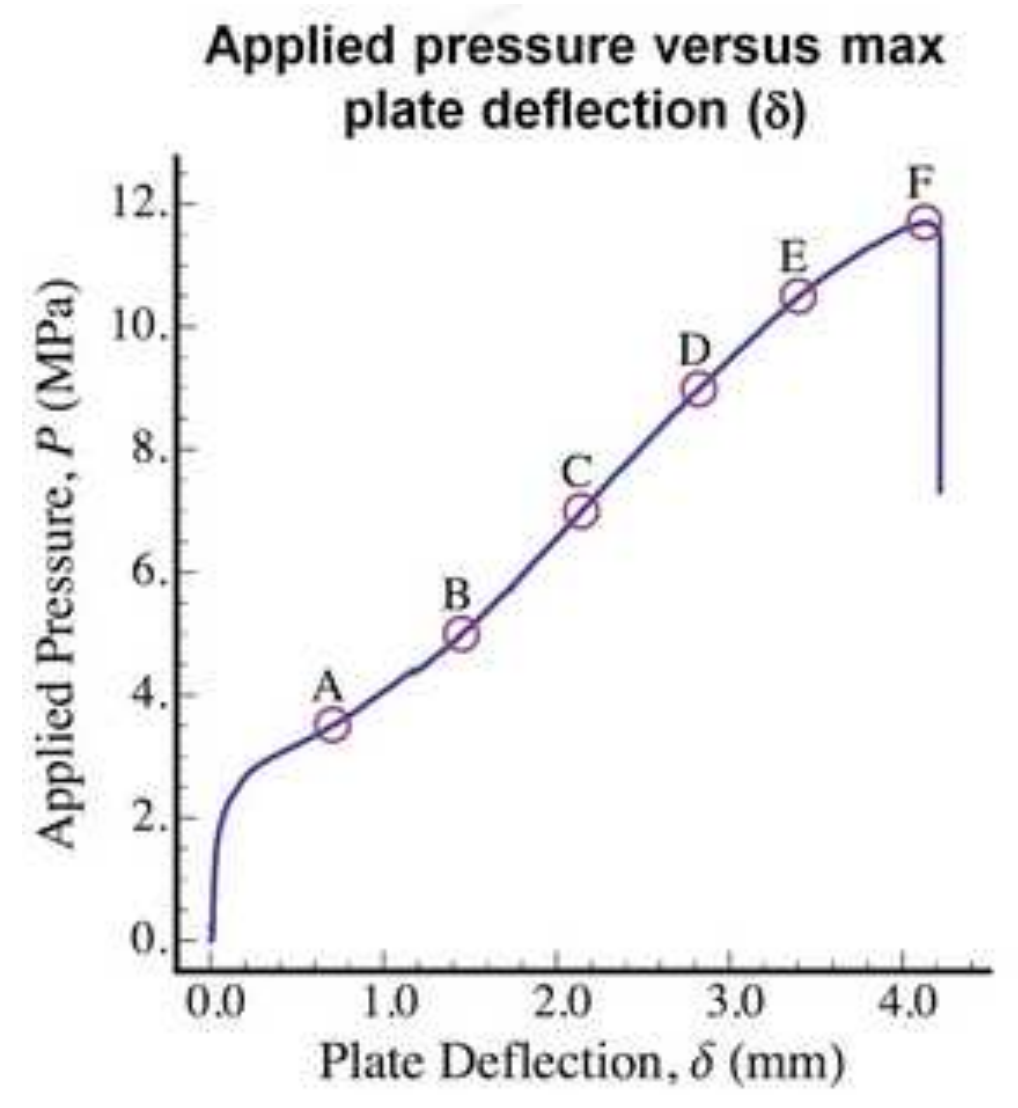

Elevation $(w)$ of the bulge at any given location

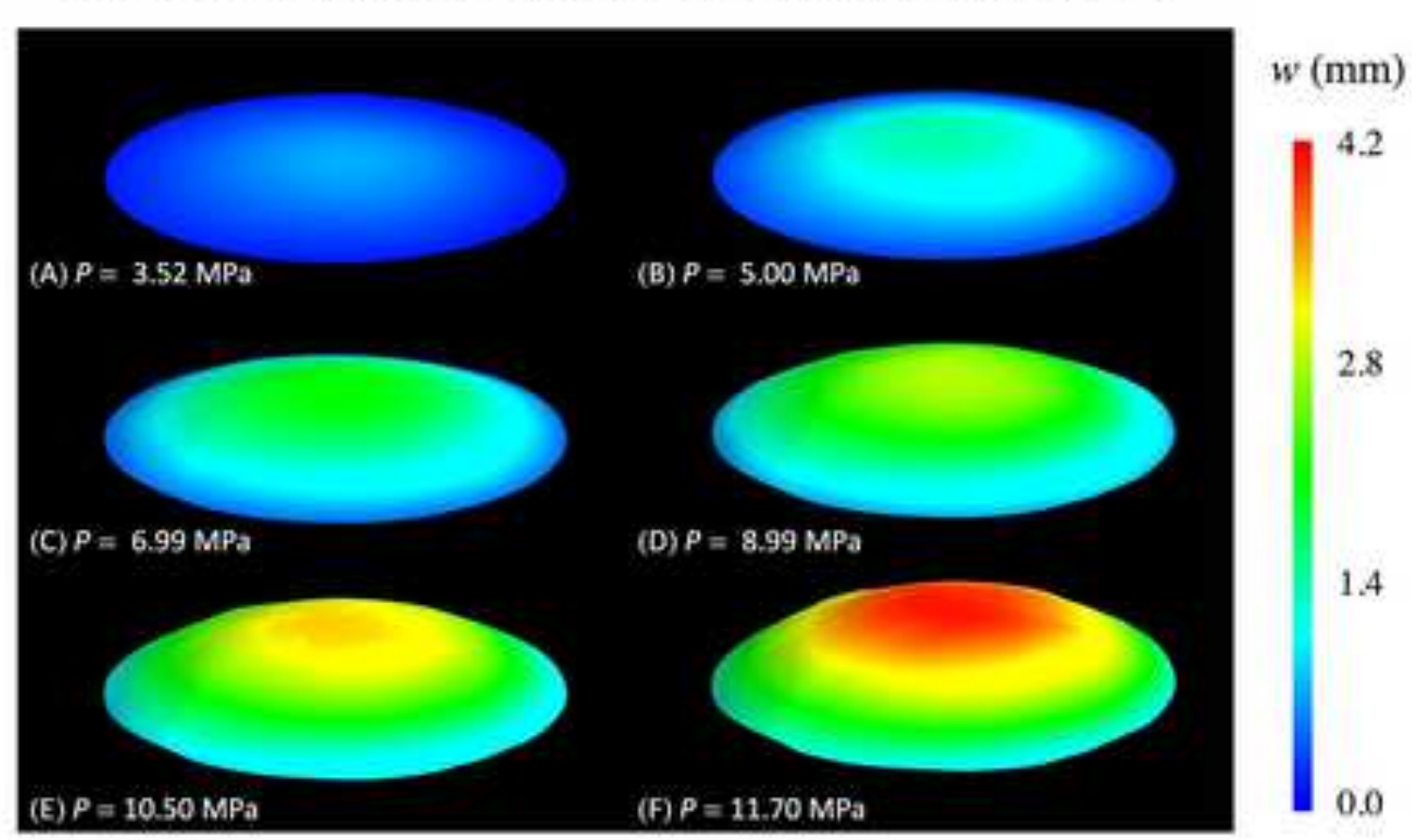




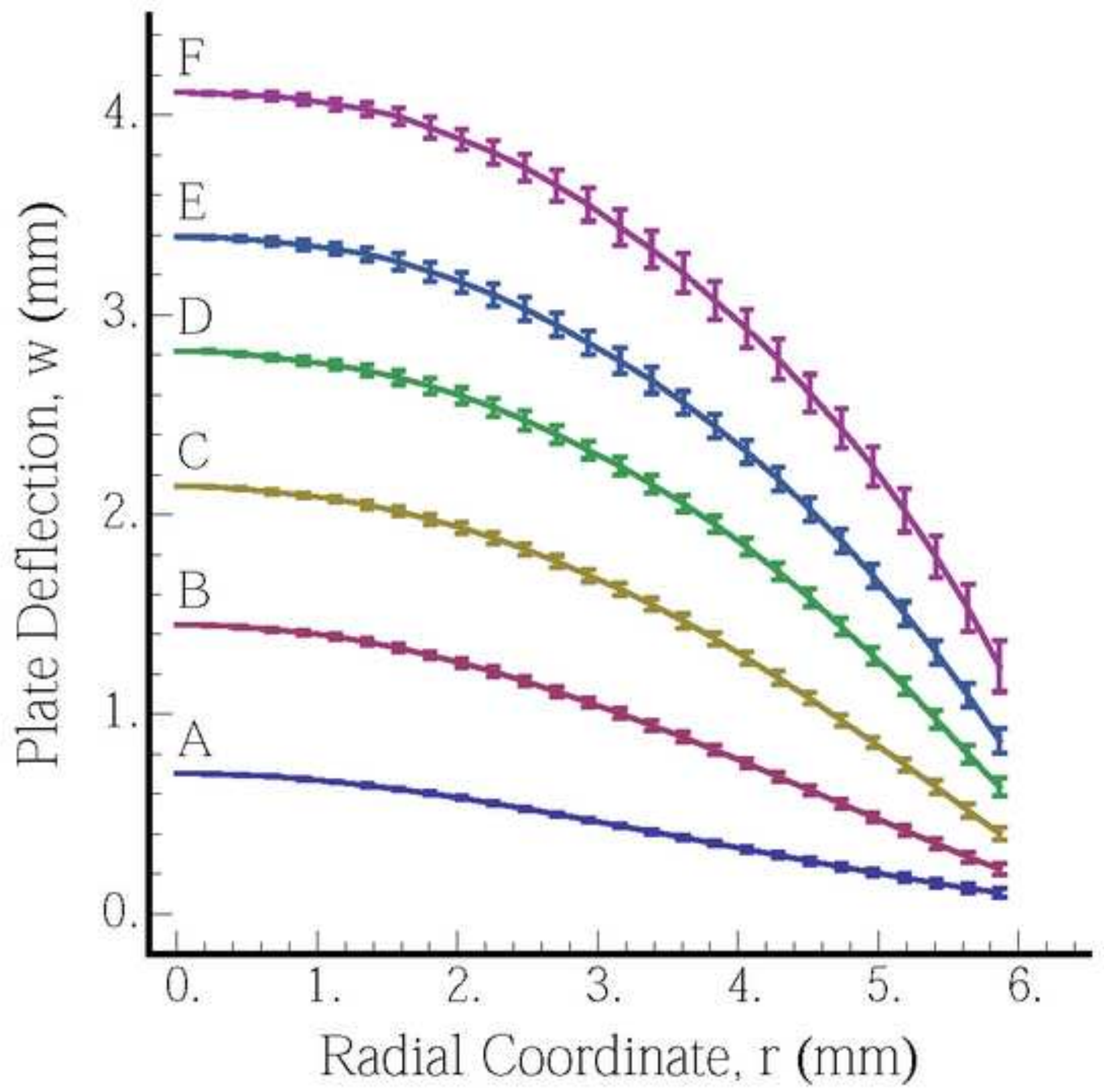



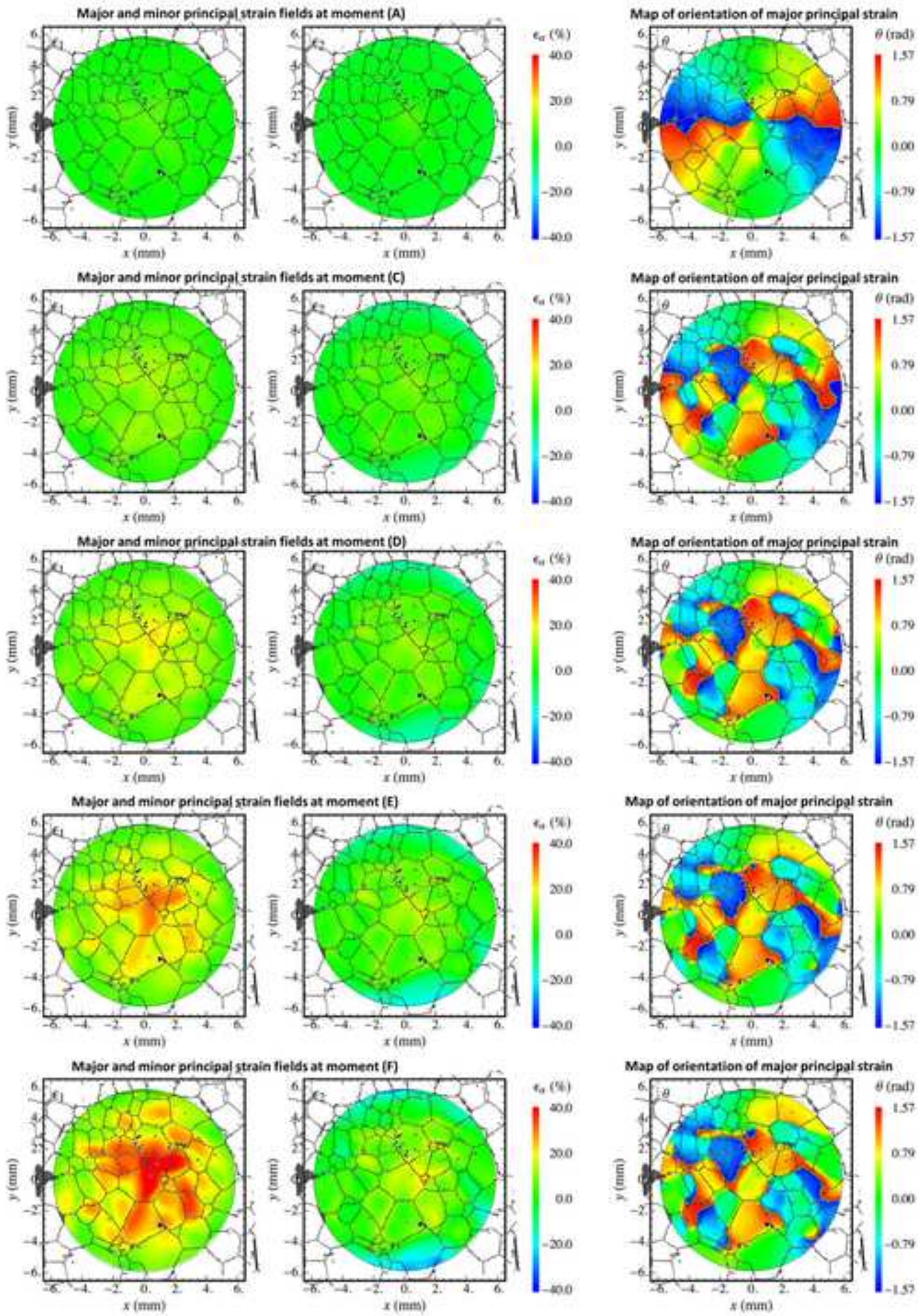

Map of erientation of major peincipal strain

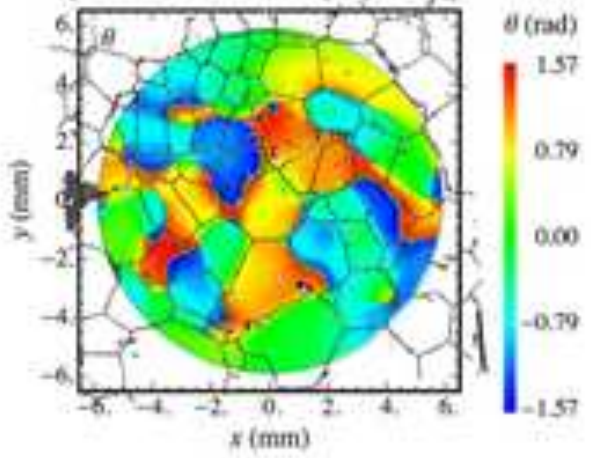

Map of ceientation of major princlpal strain

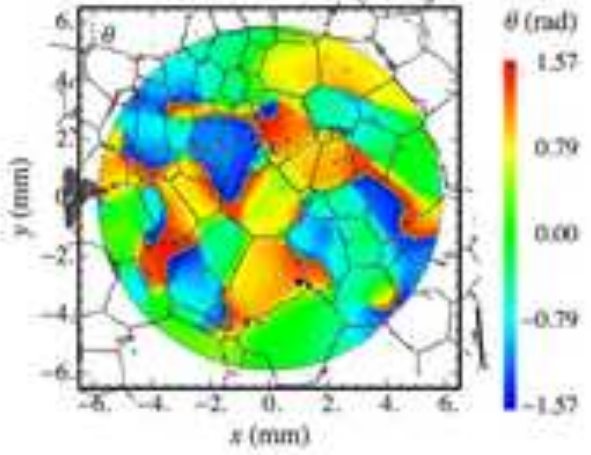



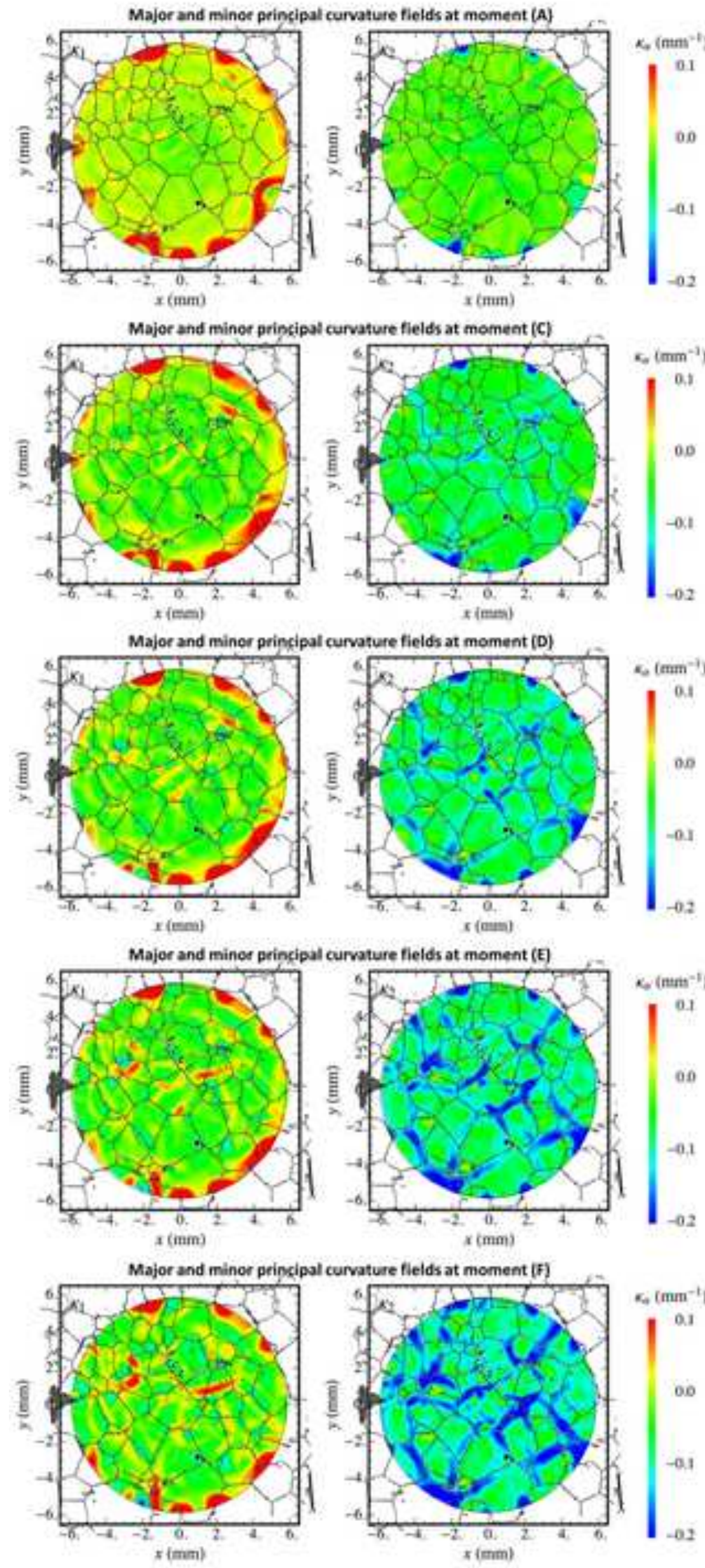

sinm $\left(\mathrm{mm}^{-1}\right)$

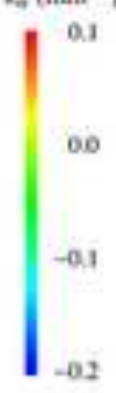

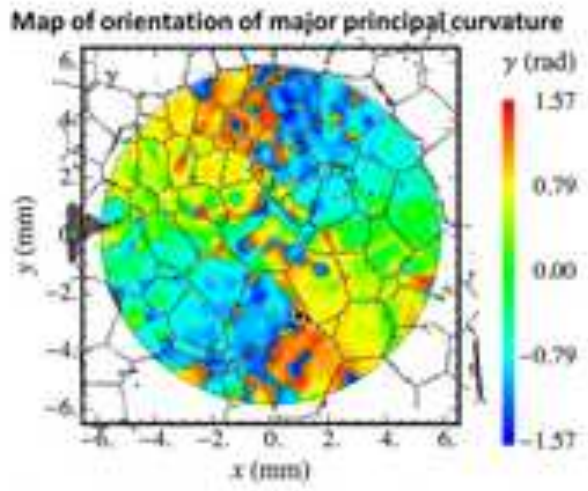

Map of erientation of majoe principal curvature

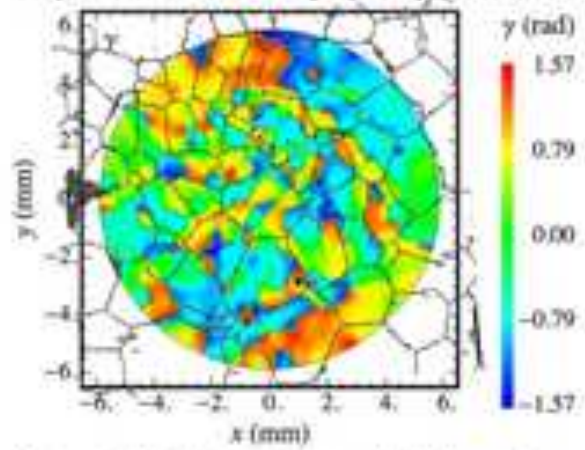

Map of orientation of majoe principal curvature

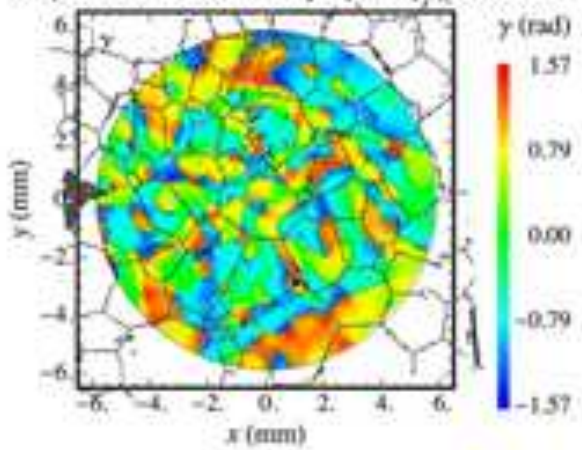

Map of orientation of major principal curvature

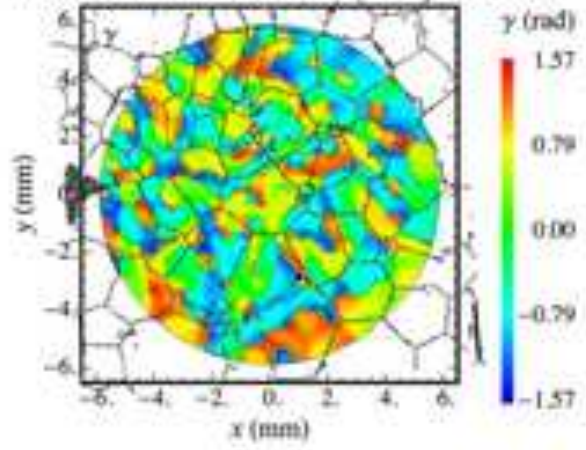

Map of etientation of major principal curvature

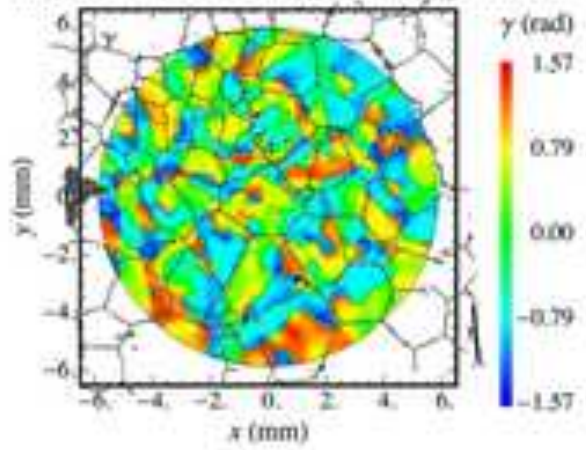



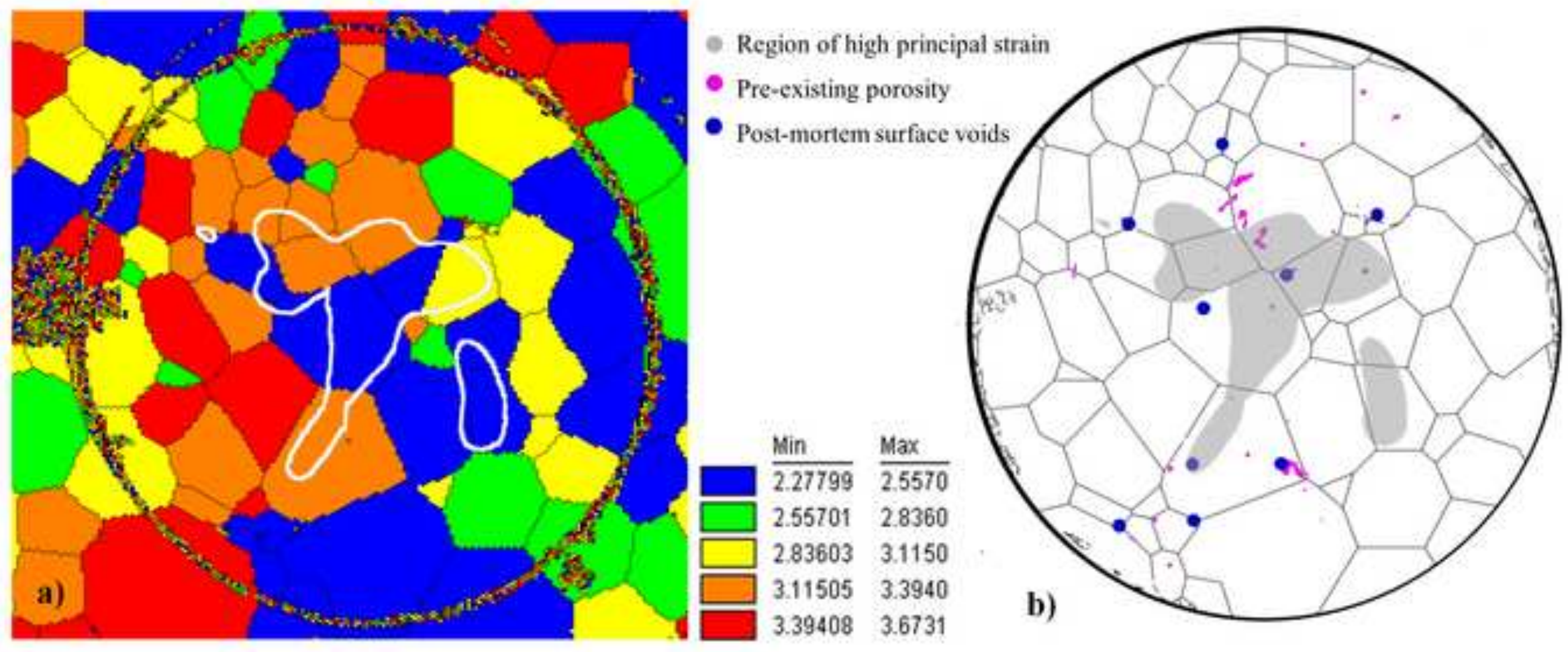

$3.39408 \quad 3.6731$

b) 